\title{
A simulated rugby match protocol induces physiological fatigue without decreased individual scrummaging performance
}

\author{
A Green, ${ }^{1} \mathrm{PhD}, \mathbf{S}$ Kerr, ${ }^{1} \mathrm{PhD}$, B Olivier, ${ }^{2} \mathrm{PhD}$, $\mathbf{R}$ Meiring, ${ }^{1}$ \\ $\mathrm{PhD}$, C Dafkin, ${ }^{1} \mathrm{MSc}$, W McKinon, ${ }^{1} \mathrm{PhD}$ \\ ${ }^{1}$ Movement Physiology Research Laboratory, School of Physiology, \\ University of the Witwatersrand, South Africa \\ ${ }^{2}$ Wits Physiotherapy Movement Analysis Laboratory, School of Therapeutic \\ Sciences, University of the Witwatersrand, South Africa
}

Corresponding author: A Green (andrewcraiggreen@gmail.com)

Background: A rugby union game consists of 80 minutes of strenuous exertion. Forwards are required to participate in the arduous activity of scrummaging throughout a game.

Objectives: The purpose of this study was to identify whether rugby-match simulated fatigue modified individual scrummaging technique and reduced performance.

Methods: Twelve forwards (body mass $106.2 \pm 13.3 \mathrm{~kg}$; stature $179.5 \pm 8.4 \mathrm{~cm}$ ) had individual scrum kinetics and kinematics assessed prior to and following a protocol that simulated a rugby match. The simulated rugby match protocol required participants to run at various velocities and perform rugby specific tasks. Rating of Perceived Exertion (RPE) was assessed using a 6-20 Borg scale and Visual Analogue Scale (VAS). Blood lactate, heart rate and RPE were measured prior to, at mid-point and after the simulated game, while markers of muscle damage (blood creatine kinase activity (CK) and urea) were measured prior to and following the protocol.

Results: RPE $(p<0.0001)$ and VAS $(p<0.0001)$ showed significant increases between the pre- and post-simulation values. Of the physiological markers, heart rate $(p<0.0001)$ and blood urea concentration $(\mathrm{p}=0.004)$ increased following the match simulation. No significant differences were observed for blood CK $(\mathrm{p}=0.281)$, individual scrummaging forces $(\mathrm{p}=0.433)$ or in the kinematic variables following the protocol. While physiological fatigue and subjective ratings of physiological fatigue may develop during a rugby simulation, no differences were observed in peak forces or in body kinematics at peak force.

Conclusion: Physiological fatigue does not influence individual scrummaging performance and technique.

Keywords: exertion, kinetics, kinematics, simulation

\section{S Afr J Sports Med 2017;29:1-6. DOI: 10.17159/2078-516X/2017/v29i0a1701}

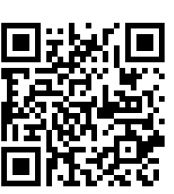

The game of rugby is a high-impact sport where bouts of considerable high-intensity effort are interspersed with periods of explosive sprints, active recovery and passive recovery. ${ }^{[1]}$ Within a competitive match, a player can cover a distance of more than $5000 \mathrm{~m}$ at various velocities. ${ }^{[1]}$ This exertion requires players to have a high level of endurance fitness and efficient physiological mechanisms to endure highintensity collisions and maintain effective technique in all the specialised aspects of the game. Both psychological [2] and physiological, ${ }^{[2-5]}$ markers of fatigue have been shown to increase following competitive rugby games. Specifically, an increase in words associated with mental fatigue, selected from the Profile of Mental State, has been reported upon match completion. [2] Regarding the objective measures of the physiological manifestation of fatigue, increased concentrations of blood metabolites, including blood urea [2] and blood creatine kinase activity, ${ }^{[2-5]}$ which indicate change in metabolism and muscle damage respectively, have been previously used to identify the metabolic effects of fatigue.

The individual demands of playing rugby are related to the players' positions, ${ }^{[1]}$ with backs required to participate in dynamic endurance events compared to the forwards' moderately static, high intensity activities. ${ }^{11]}$ The scrum is a specific and major component to the game of rugby union. A scrum requires eight players (forwards) from each team to bind together to compete in a physical push for possession of the ball. ${ }^{[6]}$ Although much of the dynamics of the force production in a scrum as a whole is yet to be revealed, the production of individual scrummaging force is thought to be related to anthropometrical, ${ }^{[6]}$ physiological ${ }^{[6,7]}$ as well as biomechanical factors. ${ }^{[8]}$

Specific assessment of physiological factors related to individual scrummaging force production has been previously related to anaerobic power ${ }^{[6]}$ and the isometric strength of the legs and back. ${ }^{[6,7]}$ An electromyographic evaluation reported that the quadriceps which lower back muscle activity ${ }^{[7]}$ are the biggest contributors to scrummaging force production.

The purpose of this study was therefore to identify the effect of simulated fatigue on an individual's scrummaging technique and performance. It was hypothesised firstly, that individual scrummaging forces would decline as a result of the match simulation, and secondly, that individual scrummaging kinematics would be negatively affected.

\section{Methods}

\section{Participants}

Twelve university-level playing forwards (body mass $106.2 \pm 13.3 \mathrm{~kg}$, stature $179.5 \pm 8.4 \mathrm{~cm}$ ) had individual scrum kinetics and kinematics measured prior to and following a simulated rugby match protocol. Study approval was given by the Human Research Ethics Committee of the University of the Witwatersrand (M131019) and written informed consent was obtained from the participants prior to the study.

\section{Study design}

The study was a simulation design, conducted in the preseason (four weeks prior to the start of the inter-varsity tournament), with the squad training consisting of eight field sessions and four gym sessions per week. The match simulation was performed following the midweek rest day, replacing one of the week's gym sessions.

\section{Individual scrummaging kinetics and kinematics}

Individual scrummaging forces were collected using an individual scrummaging ergometer at $160 \mathrm{~Hz}$. The accuracy of the ergometer has been reported, with good agreement in mass 
loading and centre of pressure location determination. ${ }^{[9]}$ The vertical and horizontal centre of pressure positions of the peak force and the scalar distance of the peak force from the centre of the scrum ergometer were calculated. All kinematics were collected simultaneously to the individual scrummaging kinetics using an 18 camera system recording at $100 \mathrm{~Hz}$ [Optitrack flex:V100r2 (Natural Point Inc., Corvallis, Oregon, USA)] using AMASS software (C-Motion Germantown, Maryland, USA). A measurement volume of approximately 12 $\mathrm{m}^{3}$ was calibrated around the scrummaging ergometer, in the area of scrummaging using a conventional wand method, until submillimetre error was established. Custom written algorithms were used to analyse body positions as derived from raw marker location data in MatLab 7 (Mathworks, Natick, Massachusetts, USA).

\section{Subjective ratings of physiological fatigue}

The individual's rating of perceived exertion (RPE) and subjective rating of physiological fatigue were collected using a 6-20 Borg scale [10] and a $100 \mathrm{~mm}$ Visual Analogue Scale (VAS), respectively. The VAS used the terms 'not exhausted (well-rested)' as the lower anchor and 'most exhausted ever experienced' as the upper anchor. Data for both ratings of physiological fatigue were collected prior to, at half-time and following the simulated rugby match protocol.

\section{Objective measures of physiological fatigue}

Heart rate was measured electrocardiographically using a Powerlab 26T (ADI instruments, 26T, Australia) and calculated as the number of $\mathrm{R}-\mathrm{R}$ intervals within one minute. Surface electrocardiograph electrodes were placed on the wrists bilaterally and grounded on the right calf muscles. Blood samples were collected via a finger prick using a spring-loaded lance (Soft-Clix pro, Roche Basel Switzerland). All samples were analysed immediately following their collection. Blood lactate was analysed using a portable blood lactate meter (Lactate Pro 2, Arkray, Kyoto, Japan). Blood creatine kinase activity (CK) and urea were analysed using a Reflotron (Roche, Roche Basel Switzerland). The Reflotron was calibrated using the manufacturer's specified procedure. Body mass was measured using a standardised digital scale (Tanita BC1000plus, Tanita Corporation of America Inc, USA). Height was measure using a standardised stadiometer.

\section{Procedure}

Participants undertook a guided warm-up that included active and passive stretching and self-determined submaximal sprints (40-60\% maximum) as they would prior to a match. Additionally, they went through one cycle of the simulated rugby match protocol to familiarise themselves with the procedures.

The simulated rugby match protocol was based on the Bath University Rugby Shuttle Test (BURST). ${ }^{[1]}$ This rugby match simulation requires players to run at various speeds, and perform tasks specific to an 80-minute rugby union game: rucks, mauls and scrums (Table 1 ).

Minor alterations were made to the BURST; two participants were tested simultaneously (ran the same cycles in the allotted time) and acted as opposition in the rugby tasks detailed below. Secondly, the number of physical collisions between the two simultaneously tested individuals were safely increased. Rucks were performed using a tackle shield (Gilbert, Grays of Cambridge (Int) Ltd, East Sussex, United Kingdom) which the defender held while the attacking player completed the tackle, remained on his feet without releasing the defender, and maintained a steady leg drive for five metres. Mauls were statically simulated and required the attacker to gain possession of the rugby ball by wrestling it from the defender. The defender was required to retain ball position for up to seven seconds. Scrums were performed one-on-one with a passive binding engagement procedure (shoulder to shoulder), where the defending player was instructed to actively resist the attacking player, while the attacking player attempted to maximally push the defender backwards for five metres. Players would alternate between attacking and defending during each task unit. The participants had a halftime break of 10 minutes following the eighth cycle, during which they were given $500 \mathrm{ml}$ of water.

Individual scrummaging kinetics and kinematics, subjective measures of physiological fatigue (VAS and RPE), heart rate and blood urea, lactate and CK concentrations were analysed before and within ten minutes after the match simulation protocol. In addition, blood lactate, VAS and RPE and heart rate were collected at half-time.

Table 1. Tasks performed during the 16 individual cycle units of the match simulation.

\begin{tabular}{lcc}
\hline \multicolumn{1}{c}{ Cycle unit tasks* } & Distance & Speed \\
\hline 1. Forward walk & $20 \mathrm{~m}$ & $20 \%$ maximal sprint \\
2. Stop and turn & - & - \\
$\begin{array}{l}\text { 3. Cruise } \\
\text { 4. Stop and turn }\end{array}$ & $20 \mathrm{~m}$ & $60 \%$ maximal sprint \\
5. Jog & - & - \\
$\begin{array}{l}\text { 6. Rugby task competed } \\
\text { against opponent }+\end{array}$ & $10 \mathrm{~m}$ & $40 \%$ maximal sprint \\
$\begin{array}{l}\text { 7. Backwards jog } \\
\text { * Cycle units were repeated 16 times; + Simulated ruck, maul or scrum }\end{array}$
\end{tabular}

\section{Data reduction}

Scrummaging back flexion was calculated as the degree of flexion between the lower and middle back around the tenth thoracic vertebra (Fig. 1). The angles of the hips were calculated as the angle between the back vector (mid-hip joint centre to mid-scapula) and the femur vector (hip joint centre to knee joint centre). Bilateral knee and ankle flexions were calculated as the angle of flexion between the vectors of the femur and the shank (knee joint centre to ankle joint centre), and the angle of flexion between the vectors of the shank and the foot (ankle joint centre to toe), respectively. Angles of $180^{\circ}$ indicated a straight back and full extension of the hip and knee, respectively.

\section{Statistical analysis}

All data were tested for normality (Shapiro-Wilk test). All normally distributed variables are represented as mean \pm standard deviations. The VAS scores and heart rates were analysed using a one-way repeated-measures ANOVA. Body mass, blood CK, urea, and individual scrummaging kinetics 
and kinematics were assessed using paired t-tests. The distributions of blood lactate and RPE values are represented as median \pm interquartile range and were assessed using a Friedman test. All statistical analyses were performed in GraphPad Prism 5 (GraphPad, San Diego, California, USA) using a significance level $p<0.05$. Effect sizes [12] were determined using Cohen's $\mathrm{d}$ and interpreted using: trivial $0 \leq \mathrm{d}<0.2$; small $0.2 \leq \mathrm{d}<0.6$; moderate $0.6 \leq \mathrm{d}<1.2$; large $1.2 \leq \mathrm{d}<2.0$; very large $2.0 \leq \mathrm{d}<4.0$.

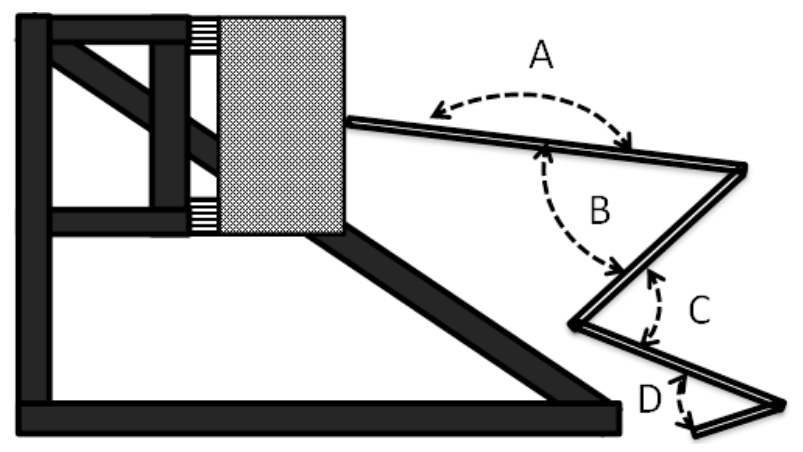

Fig. 1. Kinematic definitions of variables in the starting position from the sagittal view. All variables are calculated bilaterally. A. back flexion, B. bilateral hip extension, C. bilateral knee flexion, $D$. bilateral ankle flexion.

\section{Results}

\section{Subjective ratings and objective measures of physiological fatigue}

The RPE measured using the 6-20 Borg scale (Table 2) increased between the start and half-time $(\mathrm{d}=2.3)$ and between the start and end of the match simulation $(d=3.6)$. No significant difference was observed in the RPE between the half-time point and the end point $(\mathrm{d}=1.0)$. The second subjective marker for physiological fatigue, the VAS, increased significantly between the start and half-time $(\mathrm{d}=0.98)$, the start and end $(\mathrm{d}=1.9)$, and half-time and end $(\mathrm{d}=0.7)$ of the match simulation. Heart rates were shown to significantly increase between the start and half-time $(\mathrm{d}=3.4)$ and start and end $(d=4.0)$ of the match simulation, but not increase between the half-time and end points $(\mathrm{d}=0.3)$.

Assessing the objective physiological fatigue markers (Table 2), blood lactate was significantly higher at half-time compared to the start $(\mathrm{d}=1.0)$, but no significant difference was reported between the start and end $(\mathrm{d}=0.7)$ or between the half-time point and end point $(\mathrm{d}=0.2)$ of the match simulation. There was no significant difference in $\mathrm{CK}$ immediately following the simulated rugby match protocol $(\mathrm{d}=0.4)$. A significant increase in blood urea was observed following the simulated rugby match protocol $(\mathrm{d}=1.0)$. Body mass was significantly reduced from $106.2 \pm 13.3 \mathrm{~kg}$ to $105.0 \pm 12.9 \mathrm{~kg}$ following the rugby match simulation protocol $(\mathrm{p}<0.001 ; \mathrm{d}=0.1)$.

\section{Effect of fatigue on scrummaging kinetics and kinematics} The force magnitudes, peak force centre of pressure positions and peak force position distance from centre are reported in Table 3. The paired t-tests showed that individual peak forces, positional distance from the centre of the ergometer, horizontal or vertical force positions were not significantly different following the simulated rugby match. Effect sizes of the kinetic measurements are considered to be trivial.

There were no significant differences in the lower limb and back kinematics following the simulated rugby match protocol (Table 3). Effect sizes following the simulated rugby match protocol are considered to be within the range from trivial to small for the lower limb and back kinematics.

\section{Discussion}

It was expected that individual scrummaging forces would decline as a result of the match simulation; however, no significant differences were observed in the scrum kinetic parameters. Secondly it was hypothesised that the individual scrummaging technique (kinematics) would be negatively affected following the rugby match simulation protocol. Contrary to the expectations of the authors and despite the evident physiological fatigue, no differences were observed in the kinematic variables following the match protocol.

Subjective ratings of physiological fatigue were recorded in the current study using two different subjectively reported scales, both of which showed a marked increase (very large effect size) in perceived effort. Mashiko et al. ${ }^{[2]}$ showed how a competitive rugby game can induce physical and mental fatigue. The results of the present study agreed with the former and others ${ }^{[11]}$ with ratings of perceived exertion increasing as the duration of match simulations progresses. There were progressive increases in the subjective ratings of physiological fatigue (VAS) and an increased heart rate between the start and half-time and the start and the end of the simulation. The change in heart rates corresponds with observations made in the development of the BURST. [11] While heart rates were shown to increase during the protocol, the heart rates in the current study were lower than those noted by Roberts et al. [11] namely, 158 beats per minute. These lower values for the heart rates may have been as a result from the electrocardiography used in this study (as compared to heart rate monitors, which

Table 2. The rating of perceived exertion, heart rate and blood lactate, creatine kinase activity and urea concentrations at three time points during a rugby match simulation protocol $(n=12)$

\begin{tabular}{lccc}
\hline & \multicolumn{3}{c}{ Time point collection } \\
\hline Fatigue measurements & Pre & $\begin{array}{l}\text { Half- } \\
\text { time }\end{array}$ & Post \\
\hline & $\begin{array}{c}\text { Mean } \\
( \pm \text { SD) }\end{array}$ & $\begin{array}{c}\text { Mean } \\
( \pm \text { SD) }\end{array}$ & $\begin{array}{c}\text { Mean } \\
( \pm \text { SD) }\end{array}$ \\
Borg scale rating $(\mathrm{AU})^{*}+\ddagger$ & $6.5( \pm 3)$ & $13.0( \pm 4)$ & $16.5( \pm 5)$ \\
Visual Analogue Scale & $31( \pm 26)$ & $56( \pm 24)$ & $71( \pm 21)$ \\
$(\mathrm{mm})+ \pm \S$ & & & \\
Heart rate (beats/min) $+\ddagger$ & $75( \pm 11)$ & $111( \pm 22)$ & $117( \pm 21)$ \\
Blood lactate $(\mathrm{mmol} / \mathrm{l})^{*} \dagger$ & $1.8( \pm 1.6)$ & $3.5( \pm 3.6)$ & $2.9( \pm 3.3)$ \\
Blood creatine kinase & 203.8 & N/A & 278.1 \\
activity $(\mathrm{U} / \mathrm{l})$ & $( \pm 191.7)$ & & $( \pm 212.1)$ \\
Blood urea $(\mathrm{mg} / \mathrm{dl}) \ddagger$ & $34.6( \pm 11.5)$ & N/A & $46.5( \pm 12.9)$ \\
\hline
\end{tabular}

${ }^{*}$ Borg scale rating and blood lactate concentration are represented as median \pm interquartile range

AU Arbitrary Units; N/A Not Analysed at this time point; t significantly different between pre and half time $(p<0.05)$; $\ddagger$ significantly different between pre and post $(p<0.05)$; $\S$ significantly different between half time and post $(p<0.05)$ 
Table 3. Individual peak forces, centre of pressure scalar distance from centre, horizontal centre of pressure positions and vertical centre of pressure positions and individual scrummaging kinematics before and after a rugby match simulation protocol ( $\mathrm{n}=12)$

\begin{tabular}{|c|c|c|c|c|c|}
\hline \multicolumn{6}{|c|}{ Time point collection } \\
\hline & Pre & Post & & & \\
\hline & Mean ( \pm SD) & Mean ( \pm SD) & Difference & p value & Cohen's d \\
\hline \multicolumn{6}{|l|}{ Kinetic measurements } \\
\hline Peak force $(\mathrm{N})$ & $1720( \pm 363)$ & $1679( \pm 355)$ & -40.2 & 0.43 & 0.11 \\
\hline Peak force distance from centre (m) & $0.36( \pm .08)$ & $0.37( \pm .07)$ & 0.0037 & 0.86 & 0.05 \\
\hline Peak force horizontal position (m) & $0.012( \pm .016)$ & $0.016( \pm .026)$ & 0.0042 & 0.67 & 0 \\
\hline Peak force vertical position (m) & $-0.36( \pm .08)$ & $-0.37( \pm .07)$ & -0.0032 & 0.88 & 0.04 \\
\hline \multicolumn{6}{|l|}{$\underline{\text { Kinematic measures }}$} \\
\hline Back flexion $\left({ }^{\circ}\right)$ & $169.9( \pm 6.3)$ & $170.7( \pm 5.4)$ & 0.8 & 0.91 & 0.14 \\
\hline Right hip extension $\left(^{\circ}\right)$ & $107.3( \pm 30.4)$ & $120.7( \pm 24.4)$ & 13.4 & 0.15 & 0.49 \\
\hline Left hip extension $\left({ }^{\circ}\right)$ & $98.5( \pm 35.1)$ & $113.9( \pm 33.8)$ & 15.4 & 0.25 & 0.45 \\
\hline Right knee flexion $\left({ }^{\circ}\right)$ & $54.9( \pm 11.3)$ & $46.7( \pm 22.1)$ & -8.2 & 0.3 & 0.47 \\
\hline Left knee flexion $\left(^{\circ}\right)$ & $57.2( \pm 20.9)$ & $52.2( \pm 21.6)$ & -4.9 & 0.53 & 0.23 \\
\hline Right ankle flexion $\left(^{\circ}\right)$ & $90.6( \pm 16.6)$ & $87.2( \pm 17.1)$ & -3.4 & 0.62 & 0.2 \\
\hline Left ankle flexion $\left(^{\circ}\right)$ & $87.2( \pm 19.1)$ & $90.1( \pm 11.2)$ & 2.9 & 0.51 & 0.19 \\
\hline
\end{tabular}

may include some degree of electromyographic artefact). The heart rates reported by the Roberts et al. [11] study were collected as the mean for the five minute duration throughout the simulation, whereas the heart rate measured in the current study was only collected as single short-duration measurements, with the participants seated prior to, during the half-time break and following the simulation. Further differences may be related to the fitness levels of the participants in the various studies and as a result of the modified BURST protocol used in the current study.

In the current study blood lactate was significantly increased from before exertion to half-time, but not from half-time to post exertion or pre- to post exertion. Even though lactate significantly increased from before exertion to half-time the concentration never went above the onset of the blood lactate accumulation threshold of $4 \mathrm{mmol} / \mathrm{L}$. It is likely that the simulation consisted of more endurance tasks than explosive tasks and that the intensive static components were equally dispersed by active and passive recovery intervals. Roberts et al. [11] reported higher peak blood lactate levels during the BURST (4.4-4.6 $\mathrm{mmol} / \mathrm{L})$ compared to the half-time blood lactate values in the current study. The discrepancies in the blood lactate measurements are likely a result of the timing of blood collection. The BURST study only reported peak lactate value after 20 minutes ${ }^{[11]}$ whereas the current study reported the half-time value and not as a peak value. If the peak lactate occurred prior to this time it would have been missed, giving the participants the opportunity to metabolise the lactate. Lactate ${ }^{[3]}$ and lactate dehydrogenase ${ }^{[2]}$ have been reported to be increased following a competitive rugby game Recent data from Morel et al. ${ }^{[13]}$ would dispute that the static components of rugby are solely responsible for the increased blood lactate. They reported increases in blood lactate following scrum repetitions; however, the increase appears to be progressively lower in scrummaging compared to simulated mauling and sprinting. ${ }^{[13]}$ While the match simulation may emulate the tasks performed during a rugby match, it cannot simulate the intensity of a match. It is likely that the discrepancies are related to match intensity and the number of collisions resulting in muscle trauma and muscle breakdown. Significantly higher blood lactate concentrations have been reported in contact simulations compared to non-contact simulations. ${ }^{[14]}$ However, it must be stressed that a direct comparison between contact simulations, repeated efforts and the tasks of the current study are not equally comparable and will undoubtedly affect the development of physiological fatigue.

Physical contact in a rugby game is known to cause somatic blunt trauma and results in markers of muscle damage being released into the blood. [2-4] Creatine kinase activity (an intramuscular enzyme and therefore a marker of muscle damage) is known to significantly increase in the plasma following a game of rugby. ${ }^{[2-5]}$ The increase has been attributed to the physical impacts between players, specifically in the tackle ${ }^{[3]}$ and scrum ${ }^{[4]}$ and is thought to be related to the intensity of collisions. ${ }^{[2,5]}$ However, in the current study CK did not increase following the simulated match protocol. The simulated game, while incorporating physical contact, was likely to include considerably lower impact collisions (less than those experienced during an actual game). This was to ensure player safety and was deemed safe to have live one-onone scrummaging drills instead of a scrum sled. However, rucks and mauls had to be safely simulated using a cushioned tackle shield. These safety alterations undoubtedly affected the production of CK. Furthermore, this lack of difference may be due to the physiological pathways by which CK presents in the blood. CK is released into the interstitial fluid resulting from muscle damage and is transported by the lymphatic system back into the blood for clearance. ${ }^{[4]}$ Previous studies have shown how CK normally peaks 24 hours after a rugby game. ${ }^{[3,5]}$ The lack of an obvious increase in CK would tend to suggest that substantial muscle damage may not have been the cause of the subjective fatigue observed in this study, and that metabolic fatigue (blood urea and blood lactate) may have been the cause of the subjective fatigue.

Another metabolite known to increase with a bout of 
fatiguing exercise is blood urea. [2] Greater blood urea concentrations have been reported following competitive rugby. ${ }^{[2]}$ In rugby players an increase in blood urea was reported in the forward players and was attributed to the contact and type of game play experienced by these players. ${ }^{[2]}$ Similarly the blood urea concentration was shown to significantly increase over the simulated rugby match in the present study.

The rugby match simulation protocol resulted in a reduction in body mass, similar to that seen in the BURST $(1 \mathrm{~kg}$ in both tests). ${ }^{[11]}$ The individuals' body masses are known to be related to the individual scrum force. ${ }^{[6,7]}$ Therefore it was expected that the scrummaging force would decrease. However, the reduction in body mass was considered to have a trivial effect size, mainly due to the acute loss of water and not muscle mass. Furthermore, there was no difference between the individual scrummaging forces prior or following the rugby match simulation protocol. The development of individual scrummaging force, however, is not solely reliant on the body mass of the individual, but is related to technique. ${ }^{[8]}$

The individuals managed to attain a similar peak force before and following the simulated rugby match (effect size: trivial); however, this is in contrast to the results of Morel et al. ${ }^{[13]}$ Their study showed that an increase in the number of scrum repetitions reduced the individual scrummaging forces. [13]. Jougla et al. ${ }^{[15]}$ reported no differences between the peak forces following a rugby-specific repeated sprint protocol. These two studies suggest that scrum performance is reduced by successive scrummaging attempts and not repeated sprints. It was expected that the peak scrummaging forces would be reduced following the match simulation since scrummaging is comparable with other measures of strength, ${ }^{[6]}$ which have displayed a fatigue-related decline in magnitude. Their intrinsic ability to maintain a competitive scrummaging force may be developed over years of training, or the lack in difference might be due to the different impact nature of sprinting or jumping as compared to the high impact nature of scrummaging. The unchanging nature of scrummaging technique may, however, support the former explanation.

The resulting kinematics of the lower limbs showed that the individuals used a similar body position when applying peak force, regardless of their fatigued state. No significant difference was observed in either the peak individual forces or the body kinematics at peak force and their effect size ranged from trivial to small. Known correlates of individual scrummaging peak force are the extension of the hips, knees and flexion of the ankles. ${ }^{[8]}$ Sharp et al. ${ }^{[7]}$ reasoned that the lack of relationships between muscular activities and individual peak forces during engagement were due to a similar body position and engagement pattern used by the players. Although the relationship between muscular activity and engagement forces was not investigated in the current study, the individual kinematics did not vary, possibly as a result of similar binding and individual scrummaging styles before and after the match simulation. Therefore these authors assume that the resistance to change of the scrummaging technique (kinematics) to fatigue appears to be a consistent finding, similar to those by Sharp et al. [7] However, further investigation into scrummaging technique and the development of fatigue is required.

\section{Limitations}

The current study is limited by the small sample size of amateur rugby players. Inclusion of additional markers of physiological fatigue and performance outcomes, such as vertical jump and sprint times would have been beneficial in identifying a decline in physical performance. In addition, the match simulation protocol may have been unable to accurately replicate a competitive match, particularly the number and intensity of collisions. It should also be noted that it is possible that fatigue during actual game play may differ from the fatigue of a simulated match such as performed here. Thus more direct measures of direct match-derived fatigue may be warranted to confirm these findings. Finally, greater use of the scrummaging ergometer during the match simulation could have highlighted smaller significant changes within specific parts of the match simulation.

\section{Conclusion}

While the participants were clearly in a state of fatigue, no differences were observed in the magnitude of their peak forces or in the body kinematics at peak force. The lack of difference in the peak force despite the fatigue may suggest that individual strategies for attaining peak force are resistant to fatigue but may be multifactorial and require a more in-depth analysis.

\section{Practical implications}

- Increased perceived exertion or match-simulated fatigue does not reduce individual scrummaging force.

- Technically, scrummaging performance is unaffected by match-related fatigue.

- Due to the lack of difference in technical and physical scrummaging performances, substituting scrummaging players based solely on scrummaging performance is not recommended.

Acknowledgments: The authors express their gratitude to Liz Chase, from Wits Sports Administration, for her assistance and the willing participants from the Wits rugby club. This work was supported by the National Research Foundation under Grant UID 83772; University of the Witwatersrand, Faculty of Research Committee Individual Research Grant under Grant 0012548521101512110500000000000000004992.

\section{References}

1. Quarrie KL, Hopkins WG, Anthony MJ, et al. Positional demands of international rugby union: evaluation of player actions and movements. J Sci Med Sport 2013; 16, 353-359. [doi:10.1016/j.jsams.2012.08.005]

2. Mashiko T, Umeda $T$, Nakaji $S$, et al. Position related analysis of the appearance of and relationship between post-match physical and mental fatigue in university rugby football players. Br J Sports Med 2004; 38:617-621. [doi: 10.1136/bjsm.2003.007690]

3. Takarada Y. Evaluation of muscle damage after a rugby match 
with special reference to tackle plays. Br J Sports Med 2003; 37:416-419. [doi: 10.1136/bjsm.37.5.416]

4. Smart DJ, Gill ND, Beaven CM, et al. The relationship between changes in interstitial creatine kinase and game-related impacts in rugby union. Br J Sports Med 2008; 42:198-201. [doi: 10.1136/bjsm.2007.040162]

5. McLellan CP, Lovell DI, Gass GC. Biochemical and endocrine responses to impact and collision during elite rugby league. J Strength Cond Res 2011; 25:1553-1562. [doi:10.1519/JSC.0b013e3181db9bdd]

6. Quarrie, KL, Wilson BD. Force production in the rugby union scrum. J Sports Sci 2000; 18:237-246. [doi:/10.1080/026404100364974]

7. Sharp T, Halak M, Greene A et al. An EMG assessment of Front Row Rugby Union Scrummaging. Int J Perf Anal Sport 2014;

14:225-237.

[http://www.ingentaconnect.com/content/uwic/ujpa/2014/000 00014/00000001/art00021]

8. $\mathrm{Wu} \mathrm{WL}$, Chang JJ, $\mathrm{Wu} \mathrm{JH}$, et al. An investigation of rugby scrummaging posture and individual maximum pushing force. J Strength Cond Res 2007; 21:251-258. [DOI:10.1519/R19235.1]
9. Green A, Kerr S, Dafkin C, et al. The calibration and application of an individual scrummaging ergometer. Sports Eng 2016; 19: 59-69. [doi:10.1007/s12283-015-0188-0]

10. Borg G. Perceived exertion as an indicator of somatic stress. Scandiv J Rehabil Med 1970; 2:92-98. [PMID: 5523831]

11. Roberts SP, Stokes KA, Weston L, et al. The Bath University Rugby Shuttle Test (BURST): a pilot study. Int J Sports Physiol Perform 2010; 5:64-74. [doi. org/10.1123/ijspp.5.1.64]

12. Hopkins WG. A scale of magnitudes for effect statistics: A new view of statistics.2002. Available at: http://sportsci.org/resource/stats/effectmag.html. Accessed 20 November 2015

13. Morel B, Rouffet DM, Bishop DJ, et al. Fatigue Induced by Repeated Maximal Efforts is Specific to the Rugby Task Performed. Int J Sports Sci Coach 2015; 10:11-20. [doi:org.10.1260/1747-9541.10.1.11]

14. Mullen T, Highton J, Twist C. The Internal and External Responses to a Forward-Specific Rugby League Simulation Protocol Performed With and Without Physical Contact. Int J Sport Perform Physiol 2015; 10:746-753. [doi:10.1123/ijspp.2014-0609]

15. Jougla A, Micallef JP, Mottet D. Effects of active vs. passive recovery on repeated rugby-specific exercises. J Sci Med Sport 2010; 13:350-355. [doi:10.1016/j.jsams.2009.04.004] 